NBSIR 86-3352

\title{
A Collection of Abstracts of Selected Publications Related to Quality Assurance of Chemical Measurements
}

John K. Taylor

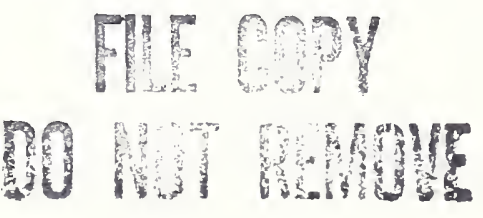

U.S. DEPARTMENT OF COMMERCE

National Bureau of Standards

National Measurement Laboratory

Center for Analytical Chemistry

Gaithersburg, MD 20899

April 1986

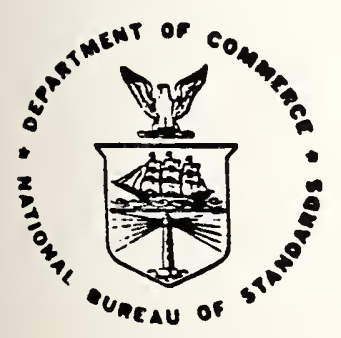

U.S. DEPARTMENT OF COMMERCE NATIONAL BUREAU OF STANDARDS 

NBSIR $86-3352$
A COLLECTION OF ABSTRACTS OF SELECTED PUBLICATIONS RELATED TO QUALITY ASSURANCE OF CHEMICAL MEASUREMENTS

John K. Taylor

U.S. DEPARTMENT OF COMMERCE National Bureau of Standards National Measurement Laboratory Center for Analytical Chemistry

Gaithersburg, MD 20899

April 1986

U.S. DEPARTMENT OF COMMERCE, Malcolm Baldrige, Secretary NATIONAL BUREAU OF STANDARDS, Ernest Ambler, Director 



\section{ABSTRACT}

This publication contains abstracts of 160 papers selected for their usefulness to analytical chemists, laboratory managers, and quality assurance officials when developing new or improving existing programs and for general guidance in producing reliable analytical chemical measurements. Definitions of some 75 terms used in describing the quality aspects of chemical measurements are included.

Key words: Accuracy of measurements; chemical analysis; chemical terminology; precision of measurements; quality assurance; quality control; reference materials. 

1. Introduction $\ldots \ldots \ldots \ldots \ldots \ldots \ldots \ldots \ldots \ldots \ldots \ldots \ldots \ldots \ldots \ldots \ldots$

2. Abstracts of Selected Papers ....................... 2

3. Terminology Used in Quality Assurance .................. 36

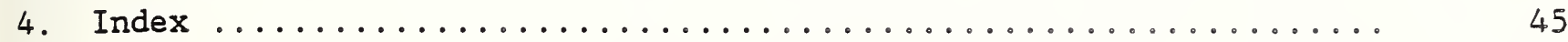





\section{INTRODUCTION}

There is a growing interest and concern for the quality of chemical measurements. Both analytical chemists and users of their services are realizing that analytical data must be both technically sound and defensible and that both objectives are best realized by following appropriate quality assurance practices developed for specific measurement situations.

Much has been written about the various aspects of quality assurance of production processes and excellent papers will be found on this subject, especially in the Journal of Quality Technology, published by the American Society for Quality Control. Less has been written about the quality assurance of measurements and especially about chemical measurements, but this situation is changing. However, the growing literature is scattered in a number of publication media.

The compilation in the present publication consists of selected papers, which, in the opinion of the author, should be especially useful when developing quality assurance programs, for the modification of existing programs, or to provide background information when considering the general principles of good chemical measurements. 
[1] ACIL -. Quality Control System .. Requirements for a Testing and Inspection Laboratory, American Council of Independent Laboratories, 1725 K St., NW, Washington, DC 20006.

A booklet describing the basic elements of QC for a testing Laboratory.

[2] American Chemical Society Committee Report, Guidelines for Data Acquisition and Data Quality Evaluation in Environmental Chemistry, Anal. Chem. 52, 2242 (1980).

A number of items are discussed that should be considered when analyzing environmental samples. Limits of uncertainty must be assigned. Full attention must be given to quality assurance.

[3] American Chemical Society Committee Report, Principles of Environmental Measurement, Anal. Chem. 55, 2210-18 (1983).

This is a revision of the "Guidelines for Data Acquisition and Data Quality Evaluation in Environmental Chemistry [Ref 2]. The original subject matter is considerably enlarged with emphasis on what is required to produce evaluated analytical data.

[4] D. M. Amsden and R. T. Amsden, Eds., QC Circles: Applications, Tools, and Theory, American Society for Quality Control, Milwaukee, WI 53203 (1976).

A collection of 23 papers on various aspects of quality circles, subdivided into the following groups: QC circle applications, USA and Japan; behavioral theory and the QA circle; QC circle tools. The papers were first presented at a conference in Toronto in 1978, sponsored by ASQC.

[5] Anon., Quality Assurance of Environmental Measurements - Information Transfer, Inc., 9300 Columbia Blvd, Silver Spring, MD 20910.

A collection of 33 papers presented at a national conference held at Denver, CO, November 27-29, 1978. The papers are classified as follows: legal and institutional problems; quality assurance methodology; water sampling and measurements; air sampling and monitoring; wastewater analysis; laboratory method control.

[6] ANSI - ASQC Standard A3-1978 Quality Systems Terminology, American National Standards Institute, 1430 Broadway New York, NY 10018

A collection of definitions of terms used in quality control and related activities. 
[7] ASTM Manual on Presentation of Data and Control Chart Analysis, STP 15D, ASTM, Philadelphia, PA 19103.

This is an excellent source of information on ways to present data by means of charts and statistical representation. The basic concepts of several kinds of Shewhart control charts are discussed with examples. Tables are provided to calculate control limits.

[8] ASTM C1009 Establishing a Quality Assurance Program for Analytical Chemistry Laboratories Within the Nuclear Industry, ASTM, Philadelphia, PA 19103.

This guide provides guidance for establishing a quality assurance program within the nuclear industry. Major topics include:

Organization; QA programs; training and qualifications; procedures; laboratory records; control of records; control of equipment and materials; control of measurements; deficiencies and corrective actions.

[9] ASTM D2777 Standard Practice for Determination of the Precision and Bias of Methods of Committee D19 on Water, ASTM, Philadelphia, PA 19103.

Detailed procedure, including statistical evaluation of data, for collaborative tests designed to evaluate analytical methodology.

[10] ASTM D3614 Evaluating Laboratories Engaged in Sampling and Analysis of Atmospheres and Emissions, ASTM, Philadelphia, PA 19103.

A detailed set of criteria which represent minimum performance standards and hence are useful for evaluating laboratories engaged in air and emissions measurements.

[11] ASTM D3856 Evaluating Laboratories Engaged in Sampling and Analysis of Water and Waste Waters, ASTM, Philadelphia, PA 19103.

A discussion of minimum requirements for laboratories engaged in water analysis of various kinds and hence useful in evaluation of such laboratories.

[12] ASTM D4210 Intralaboratory Quality Control Procedures and a Discussion on Reporting Low-level Data, ASTM, Philadelphia, PA 19103.

The various options for reporting data at or below detection levels are presented.

[13] ASTM E173 Conducting Interlaboratory Studies of Methods of Chemical Analysis of Metals, ASTM, Philadelphia, PA 19103

This standard describes procedures for sample distribution and interlaboratory testing to determine repeatability and reproducibility of methods for the chemical analysis of metals. Both graphical and numerical methods are described for analyzing the test data. 
[14] ASTM E177 Standard Recommended Practice for Use of the Terms Precision and Accuracy as Applied to Measurement of a Property of a Material, ASTM, Philadelphia, PA 19103.

This document outlines some general concepts regarding the terms "precision" and "accuracy". Notation for use in statements of precision and accuracy of measurement methodology is recommended. Precisions always should be used with descriptors to indicate causes. Accuracy depends on both precision and bias, and each component of inaccuracy needs to be specified when describing the performance of methodology.

[15] ASTM E178 Standard Recommended Practice for Dealing with Outlying Observations, ASTM, Philadelphia, PA 19103.

A general discussion of the statistical criteria for identification and rejection of outlying data. The text contains examples and tables useful in making statistical judgments.

[16] ASTM E305-83 Standard Practice for Establishing and Controlling Spectrochemical Analytical Curves, ASTM, Philadelphia, PA 19103.

Systematic and random errors that occur in obtaining data are reviewed. Background corrections are considered for linear and logarithmic readout systems. Calibration procedures are given, including the reference materials to be used and the generation of data. Procedures are provided for constructing the analytical curve, fitting the regression curve and evaluating curve fit. Control of curve shift and notation is described.

[17] ASTM E548 Standard Recommended Practice for Generic Criteria for Use in Evaluation of Testing and/or Inspection Agencies, ASTM, Philadelphia, PA 19103.

This practice provides a guide for the information needed to evaluate the technical competence of a laboratory. It may be used by accreditors or by laboratories themselves for self-appraisal.

[18] ASTM E691 Practice for Conducting an Interlaboratory Test Program to Determine the Precision of Test Methods, ASTM, Philadelphia, PA 19103.

This is the basic practice, developed by ASTM Committee E-11 on Statistical Methods, to provide guidance for the conduct of collaborative testing exercises. In addition it contains extensive and detailed diagnostic procedures (tabular, graphic, and statistical) for investigating variability in test data.

[19] ASTM E748 Quality Assurance Procedure for Spectrographic Laboratories, ASTM, Philadelphia, PA 19103.

This guide outlines the requirements for a system of quality assurance for the spectrochemical laboratory. It does not include a detailed description for setting up such a program. 
[20] ASTM E882 Accountability and Quality Control in the Chemical Analysis Laboratory, ASTM, Philadelphia, PA 19103.

This standard guide, prepared by ASTM Committee E-3 on Chemical Analysis of Metals describes approaches to be used by management in establishing a system of documented records to assure users of services that a specified level of precision is achieved in routine performance and that the data reported were obtained from the samples submitted.

[21] A. J. Barnard, Jr., R. M. Mitchell, and G. E. Wolf, Good Analytical Practices in Quality Control, Analytical Chemistry 50 (No. 12) 1079A 86A (1978).

This paper emphasizes that a laboratory must utilize good laboratory practices as a pre-requisite for the more formal aspects of a QA program.

[22] B. C. Belanger, Traceability: An Evolving Concept, Standardization News, Vol. 8, P. 22 (1980), ASTM Philadelphia, PA 19103.

A general discussion of the traceability concept - mostly from a calibration point of view.

[23] B. C. Belanger, Measurement Assurance Programs Part I: General Introduction, NBS SP 667-I, 1984, National Bureau of Standards, Gaithersburg, MD 20899.

A general discussion of the NBS MAP program. See also Reference 37 .

[24] G. A. Berman, Ed., Testing Laboratory Performance: Evaluation and Accreditation, NBS SP 591, NBS, Gaithersburg, MD 20899.

This book contains the texts of 60 papers presented at a National Conference on Testing Laboratory Performance Evaluation and Accreditation in 1979. The areas covered include: overview of evaluation and accreditation; evaluation technology; health services accreditation programs; accreditation systems and concepts; quality control; evaluation programs and systems; international coordination.

[25] J. M. Bervere and I. D. Macaulay, "Data are for Looking At or Quality Control Through Interpretation:" In Water Quality Parameters, pp. 550 562, ASTM STP 573, ASTM Philadelphia, PA 19103.

A logical interpretation procedure is described to eliminate errors, detect and evaluate anomalies, and to assure that data are valid. 
[26] D. H. Besterfield, Quality Control, Prentice-Hall (1979).

A textbook covering: Introduction to quality; fundamentals of statistics; control charts for variables; fundamentals of probability acceptance sampling; product liability; computer and quality control. Industrial slant.

[27] J. Bittner, et al., (Committee Report) Provisional Recommendations on Quality Control in Clinical Chemistry, Part 1: General Principles and Terminology, Clinical Chemistry 22, No. 4, pp. 532.540 (1978).

A good discussion of terminology and basic principles of quality control as related to clinical chemical measurements.

[28] R. H. Bordner, "Quality Assurance for Microbiological Analyses of Water," in Quality Assurance for Environmental Measurements, ASTM STP 867, J. K. Taylor and T. W. Stanley, Eds., American Society for Testing and Materials, Philadelphia, 1985, pp. 133-143.

The establishment of microbiological contaminant limits for water and wastewater, and the implementation of enforcement programs which require monitoring of these limits have emphasized the need to develop and practice quality assurance (QA) programs. A broad scope QA program is essential for microbiological determinations. The basic elements of an intralaboratory quality control (QC) program are described, which include controls over personnel, facilities, sampling techniques, laboratory operations, analytical procedures, and data reporting. Formal, written analytical procedures, a QA protocol, and complete QC records are indispensable to a QA program. The laboratory should participate in an interlaboratory QC program established to maintain minimal analytical and operational standards. The U.S. Environmental Protection Agency (EPA) certification program for analyzing public drinking water supplies is described as an example of a national interlaboratory program. Guidelines provided by the EPA for laboratory QA programs were outlined.

[29] R. Bordner and J. Winter, Eds., Microbiological Methods for Monitoring the Environment, EPA-600/8-78--017 (1978).

Discussed are: Analytical operations; general operations; analytical methodology; quality control; laboratory management. Water is the environment of concern.

[30] M. W. Brossman, Hoogheem, T. J., and R. C. Splinter, "Quality Assurance Project Plans - A Key to Effective Cooperative Monitoring Program," in Quality Assurance for Environmental Measurements, ASTM STP 867 , J. K. Taylor and T. W. Stanley, Eds., American Society for Testing and Materials, Philadelphia, 1985, pp. 53-61.

The use of cooperative monitoring programs, involving the comingling of data of the regulated community and the "regulators", can lead to effective utilization of resources and contribute to cooperative solutions to our pollution control programs. Such 
benefits can only be realized, however, under controls which ensure the comparability and validity of data obtained through well-defined roles and work plans. A key to this control is the Work/Quality Assurance (QA) Project Plan developed cooperatively by the type of team reflected in the authorship of this paper, namely by federal, industry, and state/municipal representatives.

A comprehensive Work/Quality Assurance Project/Work Plan Guidance document has been developed in the Office of Water, U.S. Environmental Protection Agency (EPA), which should effectively meet QA requirements for a wide range of users and types of watermonitoring tasks. This guidance document is designed to cover all aspects of water environmental measurement from field sampling through laboratory analysis and data reduction and, where applicable, computer input.

The document integrates a work plan with a QA plan eliminating the dual effort of overlapping plan developments and assuring practical incorporation of quality assurance/quality control. In addition, the combination of the work plan and QA plan provides a comprehensive basis to establish sound coordination and agreement for cooperative monitoring tasks. This guidance document is currently in pilot implementation throughout the United States.

[31] J. Bryson, et al., Bibliography on Laboratory Accreditation, NBSIR 822523 (1982), NBS, Gaithersburg, MD 20899.

This bibliography is a compilation of annotated references of published information on laboratory accreditation programs. An index facilitates search on the basis of country, subject area, and date of publication.

[32] S. H. Cadle, "Internal Consistency Checks as Part of a Data Validation Process for Chemical Constituents in Ambient Particulate Samples," in Quality Assurance for Environmental Measurements, ASTM STP 867, J. K. Taylor and T. W. Stanely, Eds., American Society for Testing and Materials, Philadelphia, 1985, pp. 89-99.

Internal consistency checks are an excellent means of validating data obtained during field studies of ambient particulate. The specific method used depends on the purpose and scope of the program. Techniques discussed include inspection of data for outliers, inter-sampler comparison, and ion balance and mass balance techniques. The use of these methods is illustrated with data collected in a variety of field programs. It is concluded that internal consistency checks should be incorporated into the quality assurance program of all field studies.

[33] J. P. Cali, Problems of Standardization in Clinical Chemistry., Bull. Wld. Hlth. Org. 48, 721-6 (1973).

If analytical results in clinical chemistry are to be made meaningful, i.e., accurate, precise, and specific, a systematic approach to their attainment is necessary. Furthermore, because 
this system is so complex in scope and the need for it is so widespread, it will require international coordination. Agreement on the units of measurement, the production and certification of standard reference materials, and the development of reference methods of demonstrated accuracy will require the support of all segments of clinical chemistry.

[34] J. P. Cali, et al., The Role of Standard Reference Materials in Measurement Systems, NBS Monograph 148 (1975), NBS, Gaithersburg, MD 20899.

This publication is a guide to the use of Standard Reference Materials (SRM's) and should be useful to all users of SRM's particularly those in countries developing national measurement systems. It is not intended to be an exhaustive description of the NBS-SRM program, but rather a review of the role SRM's play in the measurement system, how SRM's are certified, and what the certification means. To illustrate the use of SRM's, several selected industries are described in which SRM's have made significant contributions.

[35] S. Campbell and H. Scott, "Quality Assurance in Acid Precipitation Measurements," in Quality Assurance for Environmental Measurements, ASTM STP 867, J. K. Taylor and T. W. Stanley, Eds., American Society for Testing and Materials, Philadelphia, 1985, Pp. 272-283.

The growing interest in acid deposition has lead to a proliferation of laboratories engaged in such studies. High-level quality assurance (QA) procedures are required for each program to standardize the diverse measurement methods in use and to determine the validity of differences in measurements widely separated in space and time. Both in-laboratory (quality control) and external (quality assurance) procedures are required. A complete QA program for acid precipitation measurements must address program objectives; sites selection and operation; operator selection and training; sample collection, handling, and analyses; and data checking, storage, retrieval, and transmission. Objective criteria must be developed for detecting adulterated samples and invalid data. Appropriate laboratory and field blanks must be collected and analyzed. Standard techniques (sample spiking, replicate analysis of standards and samples) should ensure the reliability of analytical results. Relevant quality assurance data, including analytical detection limits, blank values, and the variability of replicate determinations, must be supplied with each data transmittal.

[36] CFR Title 21, Food and Drugs, Chapter 1, F\&DA, DPart 38, GLP's for NonClinical Laboratory Studies.

Federal quality assurance requirements for this subject. 
[37] CFR Title 40, Part 792, Toxic Substances Control. Fed. Reg. 48, No. 230, November 28, 1983, pp. 53922.

Federal quality assurance requirements for this subject.

[38] CFR Title 42, Public Health, Chapter 1, Public Health Service, Part 74, Clinical Laboratories.

Federal quality assurance requirements for this subject.

[39] C. Croarkin, Measurement Assurance Programs Part II: Development and Interpretation, NBS SP 676-II 1984.

A companion to Part I (see Ref. 21); discussion of development of MAP's; discussion of measurement error; use of a check standard; interpretation of special MAP's.

[40] P. B. Crosby, "Quality is Free", McGraw Hill Book Co., 1979.

A philosophical book on "how to manage quality -. so that it becomes a source of profit for your business". No quantitative discussion is given. Real life case histories are sprinkled throughout the book.

[41] W. B. Crummett and J. K. Taylor, Guidelines for Data Acquisition and Data Quality Evaluators in Environmental Chemistry, IUPAC Collaborative Interlaboratory Studies in Chemical Analysis, H. Egan and T. S. West, Eds., Pergamon Press, Oxford and New York (1982).

This paper is a review and critique of the "Guidelines For Data Acquisition and Data Quality Evaluation In Environmental Chemistry". The component parts of analytical chemistry are identified as planning, quality assurance, sampling, calibration and standardization, measurement, validation, and documentation. The guidelines recognize that a measure of uncertainty is associated with each of these components. Some of the key featcures of each of these components are discussed and the conclusion is drawn that for accurate environmental chemical analysis all these parameters must be carefully taken into account.

[42] L. A. Currie, Limits for Qualitative Detection and Quantitive Determination, Analytical Chemistry 40, 586-93 (1968).

The occurrence in the literature of numerous, inconsistent and limited definitions of a detection limit has led to a reexamination of the questions of signal detection and signal extraction in analytical chemistry and nuclear chemistry. Three limiting levels have been defined: $\mathrm{L}_{D}$-the "true" net signal level which may be a priori expected to lead to detection; and $L_{Q}$-the level at which the measurement precision will be satisfactory for quantitative determination. Exact defining equations as well as series of working formulae are presented both for the general analytical case and for radioactivity. The latter, assumed to be governed by 
the Poisson distribution, is treated in such a manner that accurate limits may be derived for both short-and long-lived radionuclides either in the presence or absence of interference. The principles are illustrated by simple examples of spectrophoto. metry and radioactivity, and by a more complicated example of activation analysis in which a choice must be made between alternative nuclear reactions.

[43] W. E. Deming, On Some Statistical Aids Toward Economic Production, Interface Vol. 5, No. 4, pp. 1-15, (August 1975).

Management's responsibility for quality outputs = from a statistical point of view.

[44] D. L. Dewar, Quality Circles - Answers to 100 Frequently Asked Questions (1979), Quality Circle Institute, P.O. Box Q, Red Bluff, CA 96080 .

An illustrated question and answer booklet describing the objectives, organization, and operation of quality circles .

[45] L. M. Eggenberger, "Establishing an Environmental Quality Assurance Program," in Quality Assurance for Environmental Measurements, ASTM STP 867 , J. K. Taylor and T. W. Stanley, Eds., American Society for Teseing and Materials, Philadelphia, 1985, pp. 391-406.

Specific steps leading to the establishment of an Environmental Quality Assurance Program in an analytical laboratory are enumerated and discussed. Aspects of a model Quality Assurance Program that applies fundamental principles and methods to selected laboratory instrumentation, and analytical protocols for the attainment of accurate, reliable results are reviewed. Discussion includes specific factors such as method validation, certification, sample handling, security, documentation, and continuing data assessment.

Objectives and problems areas characteristic of newly implemented programs, such as building a data base, interim control limits, reference standards, and categorizing and prioritizing prospective control areas, are included in the discussion.

[46] C. Eisenhart, Realistic Evaluation of the precision and Accuracy of Instrument Calibration Systems, Reprinted in Ref. 81.

A discussion of the statistical basis for specification of the precision of a measurement process. The need for statistical control is emphasized. Ways to express precision and accuracy are discussed. 
[47] EPA Handbook for Analytical Quality Control in Water and Waste Water Laboratories, EPA 600/4-79-019, March 1979.

One of the fundamental responsibilities of water and wastewater management is the establishment of continuing programs to insure the reliability and validity of analytical laboratory and field data gathered in water treatment and wastewater pollution control activities. This handbook is addressed to laboratory directors, leaders of field investigations, and other personnel who bear responsibility for water and wastewater data. Subject matter of the handbook is concerned primarily with quality control (QC) for chemical and biological tests and measurements. Chapters are also included on QC aspects of sampling, microbiology, biology, radiochemistry, and safety as they relate to water and wastewater pollution control. Sufficient information is offered to allow the reader to inaugurate or reinforce programs of analytical QC that emphasize early recognition, prevention, and correction of factors leading to breakdowns in the validity of water and wastewater pollution control data.

[48] EPA-Quality Assurance Handbook for Air Bollution Measurement Systems: Vol. I, Principles, EPA-600/9-76-005; Vol. II, Ambient Air Specific Methods, EPA 600/4-77-027a; Vol. III, Stationary Source Specific Methods, EPA600/4-77-027b; USEPA-ORD, EMSL Research Triangle Park, NC 27711 .

Volume I contains brief discussions of the elements of quality assurance. Expanded discussions of technical points and sample calculations are included in Appendices. Volume II contains guidelines for QA of ambient air measurement systems while Volume III contains similar information for source measurements.

[49] EPA QAMS-005/80, Interim Guidelines and Specifications for Preparing Quality Assurance Project Plans.

The USEPA requires that 14 points be addressed by contractors and the agency, itself, when developing QA plans for environmental measurement projects and programs. This document explains the rationale for this policy and gives specific guidance for preparing acceptable project plans.

[50] D. E. Erdmann and J. D. Thomas, "Quality Assurance of U.S. Geological Survey Water-Quality Field Measurements," in Quality Assurance for Environmental Measurements, ASTM STP 867, J. K. Taylor and T. W. Stanley, Eds., American Society for Testing and Materials, Philadelphia, 1985, pp. 110-115.

Each year many thousands of water-quality measurements are made in the field by personnel of the U.S. Geological Survey's Water Resources Division. To insure the accuracy of these measurements, an external quality assurance program was officially established in March 1979. The initial phase of this program was concerned with specific conductance and $\mathrm{pH}$ measurements. Reference samples are submitted semiannually to field analysts for measurement of 
these parameters with the same techniques and instruments used in the field. Both the personnel and the instruments involved in making the determinations are recorded. When the data are complete, a report defining the quality of the analytical results is prepared and circulated to appropriate District, Regional, and National offices. Field data, in addition to their immediate use, are normally placed in a national data base and are available to all hydrologists. As a result, the quality of field measurements can have a profound effect on their investigations. The quality assurance program described is designed to insure that the field data of the Water Resources Division are highly accurate.

[51] J. J. Filliben, Testing Basic Assumptions in the Measurement Process in "Validation of the Measurement Process" J. R. DeVoe Ed., ACS Symposium Series No. 63. (1977), American Chemical Society, Washington, DC 20036.

The basic assumptions are: randomness, fixed location, fixed variation, fixed distribution; various statistical tests for testing for such are described.

[52] L. C. Friedman and D. E. Erdmann, Quality Assurance Practices for the Chemical and Biological Analysis of Water and Fluvial Sediments, Book 5-Chapter A6 in "Techniques of Water Resource Investigations of the USGS (1982), U. S. Gov't. Printing Office, Washington, DC 20402.

This is part of an extensive publication of the US Geological Survey on methods for water resources investigations. It relates to the QA aspects of such investigations and deals with such matters as: evaluation of precision and bias of methods; interferences; quality assurance practices; preparation of reference samples; quality control as related to inorganic, organic and biological measurements.

[53] S. Gaft and F. D. Richards, Quality Assurance at Ford Motor Co., Central Laboratory - A Dynamic Approach to Laboratory Quality - In ASTM STP 814, ASTM, Philadelphia, PA 19103.

A system of internal audits that a laboratory may use to evaluate its quality assurance program and to assess the quality of its outputs.

[54] F. M. Garfield, Quality Assurance Principles for Analytical Laboratories, AOAC, Arlington, VA 22209 (1984).

A discussion of quality assurance from a management point of view.

[55] J.A. Glaser, et al., Trace Analysis for Waste Waters - Method Detection Limit., Environ. Sci. \& Tech. 15, 1426-35 (1981).

A procedure is described whereby the MDL (limit of detection for a complete methodology) may be evaluated. This is based upon the standard deviation at lowest level of measurement, $s_{O}$, and is defined as $M D L=3 s_{0}$. 
[56] A. Hainline, Jr., Quality Performance Before Quality Control, in Laboratory Management, p. 27 (Oct. 1974).

The author stresses the need for good laboratory practices before a QC program is effective. Seven reasons - management related. are given why QC systems fail.

[57] R. W. Handy, H. L. Crist, and T. W. Stanley, "Quality Assurance for Personal Exposure Monitoring," in Quality Assurance for Environmental Measurements, ASTM STP 867, J. K. Taylor and T. W. Stanley, Eds., American Society for Testing and Materials, Philadelphia, 1985, Pp. 284-286.

A personal exposure monitoring program has been carried out to field-test methodologies for sample collection and analysis. The basic study involved the collection of personal air, breath, and water samples. Quality assurance procedures were implemented during the sampling period and included exposing field controls and blanks and the collection of duplicates, some of which were analyzed by a reference laboratory. Daily checks on instrument performance and calibration were performed, and precision quality control charts were maintained. The analysis of performance audit samples was a major component of the quality assurance protocol. These results clearly show that background on the sampling devices adversely affect data quality and must be monitored closely.

[58] C. D. Hendrix - What Every Technologist Should Know About Experimental Design, CHEMTECH, Mar. 1979, p. 167.

The total experimental effort required in process and product development work can be greatly reduced through the use of 2-level factorial designs. This is true because a large number of variables can be thoroughly studied for little more than the cost of studying just one variable. The effect of each and every variable will be determined by comparing averages against averages so that the impact of random errors is minimized.

Statistically designed experiments were used in agricultural field trials more than fifty years ago, and have been used in industrial experimentation for 20 years. More sophisticated designs and methods of analysis have appeared since digital computers became readily available in the mid-1960s.

But the most useful ... and most widely used ... designs are the ones that are the easiest to practice. That's the 2-level factorial series.

[59] J. A. Henry and L. A. Knowler, Two-day Intensive Training Course in Elementary Statistical Quality Control - 3rd Ed., (1980) ASQC, Chicago Section, Chicago, IL.

12 chapters, mostly on control charts for acceptance sampling. This is the manual - 70 pages - used in a 2-day course offered by ASQC. 
[60] H. S. Hertz, and S. N. Chesler, Eds., Trace Organic Analysis: A New Frontier in Analytical Chemistry, NBS SP 519 (1979) National Bureau of Standards, Gaithersburg, MD 20899.

Researchers in diverse areas must currently perform critical analyses on minute quantities of organic compounds in various matrices. It was the aim of this symposium to bring together these scientists to discuss their common problems and to explore current and impending technology for organic analyses. Emphasis was placed on the total analysis, from collecting the sample through interpreting the results, rather than upon the measurement only. The Proceedings consist of a series of 12 invited papers by experts and particulary appropriate contributed papers. Topics covered in the Proceedings are as follows: Sampling and Sample Handling for Trace Organic Analysis, State-of-the-Art Analytical Systems, Analytical Techniques on the Horizon, Analysis of Nutrients, Analysis of Organic Pollutants and Their Metabolites in the Ecosystem, Analysis of Drugs in Body Fluid, Analys is of Food Toxicants, and Analysis of Hormones and Neurotransmitters.

[61] J. J. Hillman, "Certification of Local Laboratories Analyzing Drinking Water in Nonprimacy States," in Quality Assurance for Environmental Measurements, ASTM STP 867, J。K. Taylor and T.W. Stanley, Eds., American Society for Testing and Materials, Philadelphia, 1985, pp. 103-109.

This paper discusses the U.S. Environmental Protection Agency (EPA) certification procedures for certifying local laboratories analyzing drinking water for compliance with the Safe Drinking Water Act. A local laboratory is defined $s$ a nonprincipal state laboratory and may include state, county, municipal, federal, or commercial laboratories. The EPA certification program has recently been updated and clarified in the 1983 edition of the EPA certification manual. Critical elements for certification in chemistry, microbiology, and radiochemistry have been revised and include the preparation of a quality assurance (QA) plan.

Requirements to maintain certification, as well as, criteria and procedures for downgrading a laboratory's certification status are described in the manual. Technical services to be provided by EPA have been expanded. The paper briefly describes the certification program, changes that are incorporated in the new manual that will affect the certification of local laboratories, and potential future changes.

[62] A. Hubaux and G. Vos, Decision and Detection Limits for Linear Calibration Curves, Anal. Chem. 42, 849-55 (1970).

For linear calibration curves, two kinds of lower limits may be connected to the notion of confidence limits - a decision limit, the lowest signal that can be distinguished from the background and a detection limit, the content under which, a priori, any sample may erroneously be taken for the blank. From a few algebraical and computational developments, several practical rules are deduced to lower these limits. The influence of the 
[63] E. E. Hughes and J. Mandel, A Procedure for Establishing Traceability of Gas Mixtures to Certain NBS SRMs, EPA/781-0101 (1981).

A protocol is given that defines how "traceable" certified reference materials are to be prepared and intercompared with SRMs of essentially the same composition. The procedure the EPA uses to verify the claims of vendors is described.

[64] J. Stuart Hunter - Calibration and the Straight Line; Current Statistical Practices, AOAC Journal 64, 574 (1982).

The use of linear regression to fit a line to calibration data described. The statistical uncertainty of the line so fitted is discussed.

[65] ILAC Task Force E. Guidelines for the Determination of Recalibration Intervals of Testing Equipment Used in Testing Laboratories, International Organization for Legal Metrology (OIML). International Document No. 10, (1984), OIML, 11 Rue Twigot, Paris 95009, France.

Both intuitive and statistical guidelines are discussed for establishing realistic calibration schedules for measuring equipment. While slanted toward physical measurements, the guidelines should be useful in decisions on similar matters related to chemical measuring equipment.

[66] C. O. Ingamells and P. Switzer. A Proposed Sampling Constant for Use in Geochemical Analysis Talanta 20,547-67 (1973); 23263 (1974).

The problem of sampling for the analysis of heterogeneous materials is discussed. When the sample size is decreased, as in the case of subsampling, the standard deviation of sample composition increases. A sampling constant, $\mathrm{K}_{\mathrm{S}}$, is proposed which relates to weight of the analytical subsample, $w_{2}$ to the relative standard deviation, $R 8$, by the expression $K_{s}=R^{2} W$.

[67] S. L. Inhorn, Ed., Quality Assurance Practices for Health Laboratories, American Public Health Assoc., Washington, DC (1978).

The multiauthored 20 chapters in this book deal with various aspects of quality assurance for laboratories engaged in health (largely clinical) related measurements. The first 5 chapters are general and concerned with: laboratory management; certification; facilities and services; measurement evaluation; data transmission. The remaining chapters discuss specific quality assurance practices, largely in chemical measurement areas. 
[68] Kaoru Ishikawa, Guide to Quality Control, Asian Production Organization - Tokyo 1976.

A guide to quality control in industry. Excellent discussion of: how to collect data; histo-grams; cause-effect diagrams; parieto analysis; control charts; sampling; examples and problems.

[69] ISO Guide 25 - Guidelines for Assessing the Technical Competence of Testing Laboratories, American National Standards Institute, 1430 Broadway, New York, NY 10018 .

An international standard, similar to ASTM E-548 of criteria considered to be essential for quality outputs.

[70] IsO Guide 30 - Terms and Definitions Used in Connection with Reference Materials, American National Standards Institute, 1430 Broadway, New York, NY 10018.

Terms used in describing, measuring, certifying and reporting reference materials are deferred.

[71] ISO Guide 31 - Contents of Certificates of Reference Materials American National Standards Institute, 1430 Broadway, New York, NY 10018 .

The informational content of certificates issued for reference materials is specified and described in considerable detail.

[72] J. M. Juran, Quality Control Handbook 3rd Ed., McGraw Hill Book Co. 1974.

A compilation of 48 articles slanted mostly to QC of manufacturing and manufactured products.

[73] H. W. Kanare, Writing the Laboratory Notebook, American Chemical Society, Washington, D.C. 20036 (1985).

This book reviews the principles of proper scientific notekeeping. It goes beyond the mechanics of filling in the pages of a notebook and provides specific recommendations to help develop a flexible style of notekeeping that will serve in a variety of laboratory situations. Examples of notebook format and entry are included, and summaries of current notekeeping practices in some universities, industrial laboratories, and government research centers are presented. Alternatives to handwritten, bound notebooks are discussed, and comparison is made between the electronic notebook and the handwritten notebook. The hardware of notekeeping is described. Pens, paper, ink, notebook binding, and proper storage are all detailed. 
[74] G. Kateman and F. W. Pijkers, Quality Control in Analytical Chemistry, J. Wiley \& Sons, New York, N.Y. (1981).

Discusses topics such as sampling, analysis, data processing, organization, largely from a systems operation point of view.

[75] L. J. Kieffer, Ed., Calibration and Related Measurement Services of the National Bureau of Standards, NBS SP250, National Bureau of Standards, Gaithersburg, MD 20899 (1982).

This publication provides descriptions of the currently available NBS calibration services, special test services, and measurement assurance programs. In addition, each section describing specific services contains references to additional publications giving more detail about the measurement techniques and procedures used. This revised edition reflects the services available as of the first quarter of 1982. NBS Special Publication 250 was last issued in 1980. The Appendix to SP250 is reissued every 6 months (April and October). It lists current prices for the services described in this publication and the NBS points of contact (addresses and phone numbers) from whom additional information can be obtained.

[76] C. J. Kirchmer, Quality Control in Water Analysis, Envir. Sci. Tech. 17, 174A-181A (1983).

A general discussion of quality control as it is related to water analysis. Six sources of bias of measurements are discussed together with ways to minimize them. Ways to achieve interlaboratory quality control for measurement programs are considered.

[77] B. G. Kratochvil and J. K. Taylor, Sampling for Chemical Analysis, Analytical Chemistry 53, 924A (1981).

A general discussion of sampling with special emphasis on bulk materials.

[78] B.G. Kratochvil and J. K. Taylor, A Survey of the Recent Sampling Literature on Sampling for Chemical Analysis - NBS Tech Note 1153, Jan 1982, National Bureau of Standards, Gaithersburg, MD 20899.

An annotated listing containing references to various publications on sampling.

[79] B. G. Kratochvil, D. Wallace, and J. K. Taylor, Sampling for Chemical Analysis, Anal. Chem. Reviews 56, 113R (1984).

The sampling literature for the period 1975 to 1984 is reviewed and summarized in the following areas of chemical analysis: general considerations; theory; standards; mineralogy; soils; metallurgy; oil and gas; water; sediments; atmosphere; biology, agriculture and foot; clinical and medical. The review is based on 541 papers that are cited in the paper. 
[80] E. L. Kropp and D. N. Flannery, Guilty or Not Guilty - Only Your Statistician knows for Sure, The Environmental Forum, p. 23, April 1984.

"The measurement errors, inherent in most analytical processes, are particularly troublesome when the sample result is outside of compliance limits but within probable error margin." A plea for better measurements and their sane interpretation.

[81] H. H. Ku, Ed., Precision Measurement and Calibration: Statistical Concepts and Procedures, NBS SP 300, Vol. 1, National Bureau of Standards, Gaithersburg, MD 20899.

A compendium of 44 papers dealing with the statistical aspects of calibration and precision measurement.

[82] H. H. Ku, Statistical Concepts in Metrology, in Precision Measurement and Calibration [Ref. 75] pp. 296-328.

A general discussion of the statistical relations most used to interpret the quality of measurement data.

[83] P. D. LaFleur, Ed., Accuracy in Trace Analysis, Sampling, and Sample Handling, NBS SP 422, (1976) National Bureau of Standards, Gaithersburg, MD 20899.

This book is the formal report of the proceedings of the 7 th Materials Research Symposium: Accuracy in Trace Analysis. This volume contains the 98 invited and contributed papers presented at the Symposium, which treat the general question of accuracy and the problems of sampling and sample handling as well as the usually-discussed analytical methodology. Many important techniques and methods are described, and extensive references are presented to give deeper insight into the problems of obtaining accurate results in trace analytical chemistry. Accordingly, this volume should not only stimulate greater interest in research in these areas but should provide a valuable guide for everyday analytical problems.

[84] F. J. Linnig and J. Mandel, Which Measure of Precision? The Evaluation of the Precision of Analytical Methods Involving Linear Calibration Curves, Anal. Chem. 36, No. 13, 25A-32A, December (1964).

Ways to evaluate the precision of a method as contrasted to measurement data are discussed from the standpoint of replication error; scatter about calibration line; uncertainty of the calibration line.

[85] J.W. Locke, Ed., Laboratory Accreditation - Future Directions in the United States, NBS Special Publication 632, March 1982, National Bureau of Standards, Gaithersburg, MD 20899.

This is a reprint of the proceedings of a workshop held at NBS, in November 1981 to discuss various approaches to laboratory 
accreditation. It contains papers presented by various persons discussing the need for accreditation, modes of accreditation and ways to evaluate accreditors. Various international accreditation systems are described as well as the general approaches used in the USA.

[86] J. A. Lott, Laboratory Personnel: The Most Important Aspect of Quality Control, Medical Instrumentation 8, 22-25 (1974).

The quality of service provided by laboratory personnel who handle specimens or provide laboratory information depends completely on staff motivation and performance. Some training of new personnel can be done in the laboratory, but laboratories are becoming increasingly dependent on trained staff. One of the biggest problems in laboratories is human error. Laboratory instruments should be designed to minimize stress or boredom. Much more redundancy and computer checking of laboratory results is needed to reduce the frequency of laboratory error.

[87] J. Mandel, Fitting Straight Lines When Both Variables are Subject to Error, J. Qual. Tech.. 16, No. 1, pp. 1-14 (1984).

Least squares linear regression is one of the most widely used statistical techniques. Almost all textbooks or statistical methods provide the necessary formulas for the fitting process, based on the assumption that there is no error in the independent variable. How these formulas should be modified when both variables are subject to error is dealt with in detail using as an example an interlaboratory study.

[88] J. Mandel and F. J. Linnig, Study of Accuracy in Chemical Analysis Using Linear Calibration Curves, Anal. Chem. 29, $743-9$ (1957).

In situations characterized by linear calibration curves such as the relation between "found" and "added" in studies of accuracy in chemical analysis, the usual method for deriving confidence intervals for the slope and the intercept of the fitted straight line may lead to erroneous conclusions. The difficulty results from the interdependence of multiple conclusions drawn from the same data, especially when there is a strong correlation between the parameters involved. The method of joint confidence regions eliminates these difficulties and has the further advantage of allowing for the evaluation of the uncertainty of the calibration line as a whole, as well as of any values or functions of values derived from it.

[89] J. Mandel and T. Lashoff, Interpretation and Generalization of Youden's Two-Sample Diagram, J. Qual. Tech. 6, pp. 22-36 (1974).

A detailed statistical treatment of Youden's two-sample approach for identification of precision and bias. 
[90] S. A. Margolis, Ed., Reference Materials for Organic Nutrient Measurement, NBS SP 635 (1982) National Bureau of Standards, Gaithersburg, MD 20899.

Proceedings of a workshop held at NBS Oct. 23, 1980. Contains some of the papers presented plus reports of group discussion workshops on water soluble vitamins; carbohydrates; fat soluble vitamins; cholesterol; and fat.

[91] R. Mavrodineanu, J. I. Shultz, and O. Menis, Eds, Accuracy in Spectrophotometry and Luminescent Methods, NBS SP 378, National Bureau of Standards, Gaithersburg, MD 20899.

Proceedings of a conference held at NBS March 22-24, 1972. A collection of 18 papers discussing accuracy problems and standards for accurate measurements in spectrophotometry and luminescence.

[92] R. Mavrodineanu, Ed., Procedures Used at NBS to Determine Selected Trace Elements in Biological and Botanical Materials, NBS SP 492 (1977), National Bureau of Standards.

Methods fall under the following groups: Sample preparation; Neutron activation analysis; spark source mass spectrometry isotope dilution; atomic absorption and flame emission; molecular spectrophotometry; polarography. A total of 13 papers are included.

[93] A. G. McNish, Dimensions, Units, and Standards - - Physics Today 10, 1925 (1957).

A general discussion of the basis for physical measurements - - the units and standards developed to make measurements consistent.

[94] A. G. McNish, Fundamentals of measurement, Electrotechnology, Science \& Engineering Series 53 (1983).

A basic discussion of measurement and the propagation of error-as related to the fundamental physical quantities but good also for general education in metrology.

[95] W. W. Meinke, Ed., Trace Characterization, Chemical and Physical, NBS Monograph 100, National Bureau of Standards, Gaithersburg, MD 20899.

A compilation of papers presented at a symposium of the same name held at NBS in October 1966.

[96] MIL-STD-105 Sampling Procedures and Tables for Inspection by Attributes, Government Printing Office, Washington, DC 20402.

This publication contains sampling plans and tables for use in deciding on the acceptability (conformance with specifications) of lots based on the number of defective items observed in specified size of sample(s). 
[97] MIL-STD-414 Sampling Procedures and Tables for Inspection by Variables for Percent Defective, Government Printing Office, Washington, DC 20402 .

This publication contains sampling plans and tables for use in decisions of acceptability of lots with respect to non-conformance of items base on the basis of the mean and standard deviation of the sample of the measurements.

[98] W. J. Mitchel1, R. C. Rhodes, and F. F. McElroy, "Determination of Measurement Data Quality and Establishment of Achievable Goals for Environmental Measurements," in Quality Assurance for Environmental Measurements, ASTM STP 867, J. K. Taylor and T. W. Stanley, Eds., American Society for Testing and Materials, Philadelphia, 1985, pp. 41. 52.

In recognizing the need for measuring and documenting the quality of environmental measurements obtained in its monitoring projects and for setting achievable data quality goals for such projects, the Environmental Protection Agency issues a number of guidance and informational documents addressed to those needs. This paper reviews one of those documents, a comprehensive compilation of the quality of measurements actually obtained with various environmental measurement methods in all environmental media. Included are a brief discussion of the rationale and purpose of the document, limited reviews of the four designated measures of data quality (precision, accuracy, completeness, and method detection limit), and explanations of the format used to list the data quality information. Examples of actual data listings from the document are also included.

[99] T. J. Murphy, "The Role of the Analytical Blank in Accurate Trace Analysis" in Accuracy in Trace Analysis: Sampling, Sample Handling, and Analysis, NBS Special Publication 422, (1976) pp. 509-538, National Bureau of Standards, Gaithersburg, MD 20899.

This paper discusses the critical importance of reducing the analytical blank and controlling its variability. Practical suggestions are given to control the four major sources of blanks: the environment the analysis is performed in; the reagents used in the analysis; the apparatus used; the analyst performing the analysis.

[100] M. G. Natrella, Experimental Statistics, NBS Handbook 91, National Bureau of Standards, Gaithersburg, MD 20899.

A practical discussion of statistical evaluation of many kinds of measurement data. Many examples are worked out, in detail, and extensive tables are provided for the various statistical tests used in the analysis of data. 
[101] NBS, Brief History of Measurement Systems (With a Chart of the Modernized Metric System) NBS Special Publication 304A, National Bureau of Standards, Gaithersburg, MD 20899.

A general and easily understandable description of the fundamental system of units and standards for measurement.

[102] A. C. Nelson, Jr., D. W. Armentrout and T. R. Johnson, Validation of Air Monitoring Data, EPA 600/4-80-030 (1980).

A discussion of: data validation procedures; selection and implementation procedures; hypothetical examples and case studies.

[103] L. S. Nelson, Use of Range to Estimate Variability, J. Qual. Tech. 7 No 1. Jan. 1975 .

A discussion of the use of the range of a set of measurements to estimate " $\sigma$ ". A table of the $D_{2}^{*}$ factors needed for this purpose is included.

[104] W. Nelson, How to Analyze Data with Simple Plots, ASQC Basic References in Quality Control -. Statistical Techniques, American Society for Quality Control - Milwaukee, WI 53203.

A nice little book that discusses several graphical ways to look at data for to decide on its distribution.

[105] W. E. Oatess, Establishment of Accreditation Programs for Environmental Labs. Env. Sci. Tech. 12 1124-27 (1978).

The cooperation of accreditation organizations, regulatory agencies, consensus standards organizations, and the laboratory community are required to establish credible accreditation programs. The roles each should play are described.

[106] H. K. Oppermann and J. K. Taylor, State Weights and Measures Laboratories Program Handbook, NBS Handbook 143 (1985), National Bureau of Standards, Gaithersburg, MD 20899.

Contains criteria for assessing the capability of laboratories (basis for NBS/OWM Certification) and for appraisal for type testing of weights and measures devices.

[107] R. C. Paule and J. Mandel, Consensus Values and Weighing Factors, J. Research NBS, 87, 377-85 (1982).

A method is presented for the statistical analysis of sets of data which are assembled from multiple experiments. The analys is recognizes the existence of both within group and between group variabilities, and calculates appropriate weighing factors based on the observed variability for each group. The weighing factors are used to calculate a "best" consensus value from the overall experiment. The technique for obtaining the consensus value is applicable to either the determination of the weighted average 
value, or to the parameters associated with a weighted least squares regression problem. The calculations are made by using an iterative technique with a truncated Taylor series expansion. The calculations are straightforward, and are easily programmed on a desktop computer.

An examination of the observed variabilities, both within groups and between groups, leads to considerable insight into the overall experiment and greatly aids in the design of future experiments.

[108] G. R. Polvi, Y. S. To, and E. C. Lim, "Overview of the National Discharge Monitoring Report (DMR) Quality Assurance (QA) Program," in Quality Assurance for Environmental Measurements, ASTM STP 867, J. K. Taylor and T. W. Stanley, Eds., American Society for Testing and Materials, Philadelphia, 1985, pp. 189-199.

As mandated by the clean Water Act, wastewater treatment facilities are regulated under the national Pollutant Discharge Elimination System (NPDES). Direct discharges have unique NPDES permits with discharge limits and a self-monitoring requirement. The effectiveness of the NPDES Program hinges on the quality of the self-monitoring data.

The DMR QA Program evaluates the data quality from the NPDES permittees. Since 1978, the Program has helped to assure the data quality of DMRs. Under this program, all major permittees are annually sent performance samples, to be analyzed and reported the same way as required in the permits. The results of these permittee tests are compared with an established range of acceptance for each test parameter by EPA to identify potential problems with analytical or reporting procedures. EPA/State then follow-up through correspondence or on-site visits with permittees to resolve the problems.

As an overview, the history, authority, procedures, use of data, and implementation issues are reviewed.

[109] P. E. Pontius and J. M. Cameron, Realistic Uncertainties and the Mass Measurement Process, NBS Monograph 103, National Bureau of Standards, Gaithersburg, MD 20899 (1967).

This is the first publication that treats measurement as a process that can be held in a state of statistical control. The concepts involved and the statistical aspects are presented. The concept of statistical measurement control is based largely on the pioneering ideas presented in this paper.

[110] P. E. Pontius, Notes on the Fundamentals of Measurement as a Production Process, NBSIR 74-545 (1974), National Bureau of Standards, Gaithersburg, MD 20899.

The generalized concept of a measurement process is discussed together with techniques and examples for verifying the validity of the result. While some of the techniques may not be appro- 
priate for certain highly specialized measurement processes, it is felt that the concepts are applicable to practically all measurement processes, which must operate in a variety of environments, and which must accommodate a variety of materials and properties. The techniques have been invaluable in understanding the manner in which measurement processes operate in the "real" world.

[111] L. P. Provost, "Statistical Methods in Environmental Sampling" in Environmental Sampling for Hazardous Wastes, ACS Symposium Series 267, American Chemical Society, Washington, DC 20036 (1984).

This paper discusses the role that statistics can play in environmental sampling. Topics considered include: sampling models; determining sample size; allocating sampling resources; strategy for designing sampling plans. The mathematical expressions for computing these and related matters are presented. Because unique solutions are not possible in many cases, nomographs are given that permit quick evaluation of optional approaches in such matters as number os samples, number of measurements, and cost considerations.

[112] N. Radin, Quality Assurance in Clinical Chemistry Part I, Workbook Part II, Primer, USHEW, Public Health Service July 1971.

The primer discusses: Measurement variability; the analytical process: statistics, quality control; quality assurance. The workbook contains many practical problems to be solved.

[113] S. Reber, Laboratory Information Management Systems, American Laboratory, Feb. 1983, pp. 78-85.

The general problem of management of data in a large laboratory is discussed and a computerized system is described that is useful for this purpose.

[114] R. C. Rhodes and S. Hochheiser, Eds., Data Validation Conference Proceedings, EPA 600/9-79-042, Sept. 1979, EPA/EMSL, Research Triangle Park, NC 27711.

The proceedings are a record for future reference of the technical presentations made at a conference on Data Validation for Environmental Data. The conference was hosted and sponsored by the U.S. Environmental Protection Agency, Research Triangle Park, Interlaboratory Quality Assurance Coordinating Committee on November 4 , 1977, at the Research Triangle Park. Various data validation approaches and techniques were presented and are documented in this publication. A total of 18 papers are included.

[115] R. D. Robinson, D. Knab, and D. R. Perrin, An Individual Water Sample Quality Assurance Program, March (1985), Los Almos National Laboratory, Los Alamos, NM 10163.

This report documents the development and implementation of a flexible Individual Water Sample Quality Assurance (IWSQA) 
program. The management of the data obtained from the chemical characterization of a water sample is described. These data are used to calculate the IWSQA ratios used to evaluate the data for possible errors and indications of which chemical constituents should be reanalyzed before reporting. The ratios used are: cautions/anions; hardness/Ca $+\mathrm{Mg}$; total dissolved solids/defined calculated solids; conductivity/calculated conductivity. The theory, definitions, tables for calculation, and guidelines for interpretation of the ratios are given.

[116] L. B. Rogers, et al., "Recommendations for Improving the Reliability and Acceptability of Analytical Chemical Data Used for Public Purposes," Chem. Eng. New 1982, 60(23) 44.

Technical problems that relate to identifications and quantitative measurements of low concentrations of chemicals involved in various types of regulations and in clinical samples are addressed in a general way. Recommendations have been developed that should maximize the scientific credibility and general acceptability of the data, as well as their reliability, thereby reducing the controversial aspects of monitoring and/or regulating low concentrations of chemical species. Recommendations emphasize procedures applicable to interlaboratory comparisons.

[117] R. C. Sangster, Structure and Function of the National Measurement System, NBSIR 75-949 (1975), National Bureau of Standards, Gaithersburg MD 20899.

The general concept of a national measurement system is discussed, i.e., the framework in which compatible measurements can be made.

[118] H. Schock, Ed., Evaluation and Accreditation of Inspection and Test Activities, ASTM STP 814 (1981), ASTM, Philadelphia, PA 19103.

A collection of 21 papers dealing with various aspects of the following subjects: evaluation and accreditation concepts; evaluation and accreditation in government; international evaluation and accreditation.

[119] G. E. Schweitzer and J. A. Santolucito, Eds., Environmental Sampling for Hazardous Wastes, ACS Symposium Series 267 (1983), American Chemical Society, Washington, DC 20036.

This 13-chapter book documents the importance of improved approaches to environmental sampling and reviews several successful field programs including sampling for dioxin in Missouri, lead in Dallas, and cyanide in Washington. Classical and innovative approaches to using statistics in the design and interpretation of monitoring activities are presented. Included as well are welldeveloped guidelines for applying quality assurance procedures in the field. 
[120] R. W. Seward, Ed., Standard Reference Materials and Meaningful Measurement, NBS SP 408, National Bureau of Standards, Gaithersburg, MD 20899.

This book presents the proceedings of the 6th Materials Research Symposium held at the National Bureau of Standards, Oct. 29 - Nov. 2. 1973. It contains 25 papers describing national and inter. national programs for reference material development. The use of statistics, selection criteria, and steps for certifying SRM's are reviewed. Reports of 15 panel sessions reviewing the use and needs for reference materials are included.

[121] W. A. Shewhart, Statistical Method from the Viewpoint of Quality Control, The Graduate School, U. S. Department of Agriculture, Washington DC (1939).

This book contains the texts of a series of lectures on the philosophy of statistical product control. It is one of the earliest discussions of this topic that has provided the background for modern quality control.

[122] E. G. Shilling, A Lot Sensitive Sampling Plan for Compliance Testing and Acceptance Inspection, J. Qual. Tech. 10, 47.51 (1978).

An acceptance sampling plan is presented that is applicable in general acceptance sampling and is particularly useful in compliance and safety-related testing. It provides for rejection of the lot if any defective items are found in the sample; a well. defined relationship between the sampling plan the size of the lot being inspected; a clear indication of the economic impact of the quality levels utilized in the plan; and simplicity and clarity in use.

Based on the hypergeometric probability distribution, it gives the proportion of the lot that must be sampled to guarantee that the fraction defective in the lot is less than a prescribed limit with odds of 9:1 (i.e., LTPD protection). Various applications are presented; together with a discussion of the operating characteristics and other measures of the plan's protection.

[123] F. H. Squires, Successful Quality Management, Hitchcock Executive Book Service (1980).

A collection of monthly articles appearing in "Quality" Magazine. Chapter titles are: Quality Management and You; Executive's Guide to Quality; Product Liability; Employee Motivation; Effective Communication; Inspection and Testing; Statistics and Sampling. A total of 341 articles are included under the above topics.

[124] Standard Reference Materials Catalogue, NBS SP 260, National Bureau of Standards, Gaithersburg, MD 20899.

A catalogue describing the SRMs available from NBS. 
[125] T. W. Stanley and S. S. Verner, "The U.S. Environmental Protection Agency's Quality Assurance Program," in Quality Assurance for Environmental Measurements, ASTM STP 867, J. K. Taylor and T. W. Stanley, Eds., American Society for Testing and Materials, Philadelphia, 1985, pp. 12-19.

The Environmental Protection Agency (EPA) and its predecessor organizations historically have devoted considerable time and effort to the standardization of test procedures, the development and use of calibration and performance audit procedures, quality control and reference samples, training, and other quality assurance (QA) activities. However, these QA activities were essentially voluntary, and most of the data generated were reported with no indication of quality, thereby severely reducing their usefulness. Due to questions concerning the quality of EPA's data, agency policy stipulated on 30 May and 29 June 1979, requires participation in a centrally managed QA program by all organizational units engaged in environmentally related measurements. This policy applies equally to those extramural efforts performed on behalf of EPA. The primary responsibility for program development and direction is assigned to the Office of REsearch and Development, while other program offices, regions, and laboratories are responsible for its implementation.

Essential elements of the program include developing and implementing QA program plans, QA project plans, and standard operating procedures; conducting audits of the capability and performance of measurement systems, and data quality; maintaining a mechanism for corrective actions; QA training; and frequent reports to management on the quality of data, program effectiveness, and problems. The goal of the QA program is to ensure that all data generated are of known, documented, and acceptable quality.

[126] D. R. Taylor, Quality Assurance Guidance for the Determination of Inorganic Parameters in Soil, Sediment, and Water Samples, EPA, 600/X83-013, February (1983).

The purpose of this document is to describe the QA/QC procedures which should be followed by any laboratory attempting to produce analytical data of known quality from the analysis of inorganic compounds in samples of soil, sediment, water or hazardous wastes regulated under the Resource Conservation and REcovery Act (RCRA). Most of the QA/QC procedures described herein are those currently used in the National Contracts Analytical Laboratory Program. However, since the NCALP program is undergoing an evolutionary process, the analytical protocols are constantly be improved by input from EPA and contract laboratories, as well as from other sources. The performance control windows for the analytical processes described will be continually updated using a computerized analytical data base compiled at the Environmental Monitoring Systems Laboratory at Las Vegas. 
[127] J. K. Taylor, Validation of Environmental Data by Intercalibration and Laboratory Quality Control Programs. Preprint, ACS Division of Environmental Chemistry, Los Angeles CA, April 1974.

Accurate methodology, adequate calibrations, and systematic quality control procedures are required to provide reliable analytical data. These three factors may be considered as links in a chain that is only as strong as the weakest segment. Well. established measurement processes are conventionally established by deliberate or evolutionary processes but environmental analysis has weak foundations in each of the three areas. The rapid growth of this field, both in the number of analyses required and in the variety of substances that must be controlled or measured to evaluate their actual or potential environmental impact is largely responsible for this situation. Legislative requirements have often forced the acquisition of data that would have better remained unreported. The situation needs to be rectified by a systems approach that assures the validity of the data reported by individual stations and permits its incorporation into the body of data derived from diverse sources. This paper discusses the measurement system with the objective to encourage improvements where needed.

[128] J. K. Taylor, Importance of Inter-calibration in Marine Analysis, Thal. Jugo. $14221-29$ (1978).

This paper discusses the general principles of intercalibration, the process by which measurement stations may be calibrated with respect to each other. When intercalibrated, the measurements generated may be considered as mutually compatible and admissable into a body of data for compilation or other purposes. The process involves the use of recognized sampling techniques and measurement methods, as well as rigorous quality control procedures within each measurement station. Standard Reference Materials can provide the intercalibration linkage to verify that compatibility of data does exist.

[129] J. K. Taylor, Quality Assurance of Chemical Measurements, Anal. Chem. 53, 1588A-96A (1981).

This is a general article describing the basic aspects of quality assurance as related to chemical analysis.

[130] J. K. Taylor, Quality Assurance Measures for Environmental Data, in "Lead in the Marine Environment", M. Branica and Z. Konrad, Eds., Pergamon Press pp. 1-7 (1980).

An understanding of the occurrence, fate, effects, and pollution potential of suspected toxic substances requires a wide variety and large quantity of analytical data, obtained over extended time intervals, in diverse locations, and by independent investigators. Unfortunately, much of the data reported in the literature is often difficult to interpret and correlate because of lack of information of the reliability of sampling and measurement. This 
paper discusses general principles of quality control of both measurement and sampling and recommends minimum requirements for reporting environmental data. The use of generally accepted reference materials to intercalibrate measurement laboratories is emphasized.

[131] J. K. Taylor, Reference Materials -. What They Are and How They Should be Used, J. Testing and Evaluation 11, 355-7 (1983).

Reference materials consist of known samples that may be used to verify the accuracy of measurements of a system known to be in statistical control. They are useful, also, for evaluating methodology and for evaluating the proficiency of analysts/laboratories. Ways to use reference materials, systematically, are described.

[132] J. K. Taylor, Principles of Quality Assurance of Chemical Measurements, NBSIR 85-3105 National Bureau of Standards, Gaithersburg, MD 20899.

The general principles of quality assurance of chemical measurements are discussed. They may be classified as quality control - . what is done to control the quality of measurement process, and quality assessment -. what is done to evaluate the quality of the data output. Quality assurance practices are considered as a hierarchy with levels progressing from the analyst, the laboratory, the project, to the program. The activities of each level are different and depend upon the ones beneath it. Recommendations are presented for developing credible quality assurance practices at each level. An appendix contains outlines that may be used to develop the various documents associated with a quality assurance program. An extensive list of definitions of terms used in quality assurance is included.

[133] J. K. Taylor, What is Quality Assurance? - - in Quality Assurance for Environmental Measurements, ASTM STP867, J. K. Taylor and T. W. Stanley, Eds., ASTM Philadelphia, PA 19103 pp. 5-11 (1985), (See Ref. 108).

The quality of data must be known and established before it can be used logically in any application. Data quality may be judged on the basis of its quantitative accuracy and on the confidence that can be placed in the qualitative identification of the parameters measured. This requires the production of data in a quality assurance program that permits the assignment of its statistically supported limits of uncertainty. The essential features of such a program, consisting of quality control and quality assessment techniques, are discussed in the paper.

[134] J. K. Taylor, Handbook for SRM Users, NBS SP 260-100, (1985), National Bureau of Standards, Gaithersburg, MD 20899.

A comprehensive discussion of the use of reference materials to assess the accuracy of a measurement system. Also included are the selection of SRM's, the certification process, and the 
measurement of certified values. An appendix reviews the statistical procedures and statistical tables useful to evaluate results of measurements.

[135] J. K. Taylor, Validation of Analytical Methods, Anal. Chem. 55, 600A608A (1983).

Validation is defined as the process of ensuring that methodology is potentially useful for a given purpose. The hierarchy of analytical methodology is discussed as technique, method, procedure, and protocol, each of which should be validated.

[136] J.K. Taylor, Guidelines for Evaluating the Blank Correction, Journal of Testing and Evaluation 12, 54-5 (1984).

The statistical considerations in applying the blank correction in trace analysis are discussed. The question of acceptable limits for the blank is addressed. Unless sufficient measurements are made, the uncertainties in the blank correction may be the major source of uncertainty in ultra-trace analysis.

[137] J. K. Taylor, Essential Features of a Laboratory Quality Assurance Program, in Statistics in the Environmental Sciences, ASTM STP 845, S. M. Gertz and M. D. London, Eds., ASTM Philadelphia, PA 19103.

Progress in the environmental sciences is vitally dependent on reliable data resulting from complex measurement processes. Because of this complexity, the measurement process must be well designed and operate in a state of statistical control. A quality assurance program, including quality control and quality assessment procedures, denotes those features that lead to the produc. tion of data under these conditions. The rudimentary features are described together with the expected benefits. Parallelisms are drawn with a well designed manufacturing process.

[138] J. K. Taylor, Quality Assurance for a Measurement Program, in Environmental Sampling for Hazardous Wastes, G. E. Schweitzer and J. A. Santolucito, Eds., ACS Symposium Series 267, American Chemical Society, Washington, DC 20036 (1985).

Quality assurance for measurement programs requires participants of demonstrated competence, using appropriate quality assurance procedures. Responsibilities of particlpants and managers are discussed. The use of a reference laboratory to assure that program objectives are met is described and recommended.

[139] J. K. Taylor, Evaluation of Data Obtained by Reference Methods, in Calibration in Air Monitoring, ASTM STP598 (1976) ASTM, Philadelphia, PA 19103.

The key role of reference methods for measurement of air pollutants in regulatory matters demands that the data obtained in their use be precise and accurate, but no procedures have been established for evaluating its reliability and validity for the 
intended use. This paper discusses the general principles of reliable analytical measurements and presents guidelines by which the quality of data obtained by reference methods or other procedures may be evaluated.

[140] J. K. Taylor and T. W. Stanley, Eds., "Quality Assurance for Environmental Measurements", STP 867, American Society for Testing Area Materials, Philadelphia, PA 19103 (1985).

This book contains the text of 36 papers presented at a conference of the same title at the University of Colorado, Boulder $C O$ in August 1983. The contents are as follows: General Topics - 2 papers; Data Quality Assessment - 3 papers; Ambient Air Measurements - 3 papers; Ambient Water Measurements - 4 papers; Source Measurements - 4 papers; Discharge Monitoring - 6 papers; Quality Assurance Management - 2 papers.

[141] J. K. Taylor, The Quest for Quality Assurance, American Laboratory, pp. 67-75, October (1985).

The basic need for a measurement assurance program is discussed and various approaches are described. The ethical and professional responsibilities of analytical chemists are discussed and a code of ethics for the practice of analytical chemistry is presented.

[142] J. K. Taylor, The Role of Collaborative and Cooperative Studies in Evaluation of Analytical Methods, J.A.0.A.C. 69, 398 (1986).

A method proposed as a standard or for use in a regulatory process or other purposes must be reliable and its typical performance characteristics should be stated and verified. Collaborative testing is the most acceptable way to accomplish the latter but its function should not be misunderstood. Such testing can verify performance characteristics and experimentally demonstrate that the methodology can be used successfully by a representative group of laboratories. It does not necessarily support the validity of any data obtained using the method because this may depend on many other factors including the expertise use of the laboratory and the quality assurance aspects of its measurement process.

[143] N. W. Tietz, A Model Comprehensive Measurement System in Clinical Chemistry, Clin. Chem. 25/6, 833-39 (1979).

A conceptual system of employing validated methods and reference materials is discussed to provide the accuracy required of clinical measurements. Topics discussed include: definitive methods; reference materials. A measurement system for the determination of calcium in body fluids, based upon the above principles, is described. 
[144] J. Visman, A General Sampling Theory, Materials Research and Standards, November 1969, p8.

A general theory is presented for sampling, based on the premise that the variance in a bulk lot of a material can be expressed by a relation incorporating two sampling constants. The relation may be used to design sampling programs, and to determine in advance the precision of a sampling experiment as a function of sample size and the number of increments. See also discussions of this in the articles listed below:

Duncan, A. J. Comments on "A general sampling theory", Mat. Res. Stds. $11(1): 25 ; 1971$.

In this note it is shown that the empirical results of Visman can in part be derived from statistical theory.

Visman, J.; Duncan, A. J.; Lerner, M. Further discussion: "A general theory of sampling", Mat. Res. Stds. 11(8):32; 1971. In this note Visman and Duncan discuss the generality of the Visman approach, as well as its validity under certain circumstances. Lerner summarizes their points of view, and concludes that the Visman procedure, while not strictly 'general', is a giant step above the previous rules-of-thumb often used.

Visman, J.; Duncan, A. J. Discussion 3 on "A general theory of sampling". J. Mat. 7:345; 1971.

Here Visman develops further the factors that hold the constant relating to segregation variance at a stable value. Duncan agrees with Visman's treatment and conclusions, and develops the statistical theory for Visman's approach.

[145] R. J. Wening, The Role of the Quality Control Manual in the Inspection and Testing Laboratory, In NBS SP 591, PP. 99-103 (see reference [22]).

The QA manual is the cornerstone of a laboratory's credibility and an important ingredient in its assessment for accreditation. Its content and mode of use are described.

[146] G. Wernimont -. Ruggedness Evaluation of Test Procedures, ASTM Standardization News, pp. 13-16, March 1977.

A discussion of Youden's ruggedness test with extension to testing 18 variables.

[147] Western Electric - Statistical Quality Control Handbook - Select Code 700-444, Western Electric Co., Commercial Sales Clerk, P.0. Box 26205, Indianapolis, IN 46226.

This book contains a wealth of information on the applications of control charts to monitoring industrial production processes. Particularly useful is the extensive treatment of diagnosis of production problems via control charts. While written for 
engineers operating industrial processes, the extension of the ideas to measurement processes is obvious in most cases.

[148] J. D. Westgard and T. Groth, A Multi-rule Shewhart Chart for Quality Control in Clinical Chemistry, Clin. Chem. 27, 493-501 (1981).

Presents a decision chart and suggests several courses of action when interpreting control chart data.

[149] L. R. Williams, Guidelines for Conducting Collaborative Testing of Biological Test Methods, EPA 600/X-83-029, 1983.

Summarizes the approach, approved by EPA's Office of Toxic

Substances, to conduct collaborative tests of biological methods.

[150] L. R. Williams, "Quality Assurance Considerations in Conducting the Ames Test," in Quality Assurance for Environmental Measurements, ASTM STP 867, J. K. Taylor and T. W. Stanley, Eds., American Society for Testing and Materials, Philadelphia, 1985, pp. 260-271.

This paper provides general and specific guidance for the development and implementation of a quality assurance program for Ames testing. An effective quality assurance program is needed to assure that data produced from short-term mutagenicity testing (e.g., the Ames test) are of known quality and comparable among laboratories conducting the testing. The success of such a program begins with a personal and organizational commitment to data quality. Next, standardized procedures for the conduct and control of the testing process should be adopted and rigidly followed. Finally, participation in an external quality assurance program is necessary for independent confirmation of testing performance and confirmation of interlaboratory data comparability. Comparability of test data is required if there is to be confidence in the results of testing activities and acceptance of the resulting interpretation of test findings.

The functions and responsibilities of an effective quality program are outlined. Primary functions include development of standards based upon technically attainable criteria, assistance to participating laboratories in adopting those standards, and independent evaluation of the success of the laboratories in meeting those standards.

[151] A. L. Wilson, Approach for Achieving Comparable Analytical Results from a Number of Laboratories, Analyst 104, 273 (1979).

QA in a water lab is discussed. Close attention to many details is stressed.

[152] A. L. Wilson, The Performance Characteristics of Analytical Methods, I. Talanta 17, 21-29 (1970).

The ever-increasing volume of analytical literature makes it important to be able to compare unambiguously the advantages and 
disadvantages of analytical methods. To this end, a set of consistent definitions and methods for determining quantitative performance-characteristics (e.g., precision, sensitivity, bias) is needed. The aim of this series of papers is to review the definition and determination of such parameters, and to suggest criteria for general use. This first paper discusses the general problem, considers those general aspects of analytical methods that are important, and establishes the performancecharacteristics to be considered in detail.

[153] A. L. Wilson, The Performance Characteristics of Analytical Methods = II Talanta 17, 31-44 (1970).

Statements on the errors of analytical results are an important aspect of characterizing the performance of analytical methods. The general nature of random and systematic errors is briefly discussed, and methods of numerically defining the former are considered. It is suggested that the standard deviation of analytical results be used exclusively as the quantitative measure of precision within the context of performance-characteristics. Techniques for, and precautions to be observed in, estimating standard deviation are critically discussed. On this basis, general principles are proposed that should be observed whenever possible in experimental tests to estimate standard deviation.

[154] A. L. Wilson, The Performance Characteristics of Analytical Methods III Talanta 20,725-32 (1971).

The range of concentrations covered by an analytical method is an important performance-characteristic, and suggestions are made for definition of this range. The ability to detect small concentrations is also considered, and it is also suggested that the standard deviation of blank determinations be quoted as the performance-characteristic relevant to the power of detection.

[155] W. J. Youden, Collection of Papers on Statistical Treatment of Data, Journal of Quality Technology, Vol. 4, No. 1, pp. 1-67, January 1982.

This memorial issue of the Journal of Quality Technology reviews the contributions of the late W. J. Youden to the statistical treatment of measurement data. It contains a biography, selected reprints, and a complete bibliography of his publications.

[156] W. J. Youden, Experimentation and Measurement, NBS SP 672, 1984 , National Bureau of Standards, Gaithersburg, MD 20899.

Reprint of a book originally published by the National Science Teachers Association. A very readable primer written for the high school student level to introduce one to statistical ideas with practical examples. 
[157] W. J. Youden, Ranking Laboratories by Round Robin Tests, Materials Research and Standards, January 1963.

A procedure is described to evaluate the performance of laboratories by ranking them according to the magnitude of the results they report on a series of test samples. A statistical test to recognize laboratories that consistently report low and high results is given.

[158] W. J. Youden, Statistical Techniques for Collaborative Tests, AOAC, Arlington, VA 22209.

The philosophy of collaborative testing is discussed together with the statistical evaluation of test data. This is the predecessor of the publication in Reference 149, which also should be consulted in this regard.

[159] W. J. Youden and E. H. Steiner, STatistical Manual of the AOAC, AOAC, Arlington, VA 22209, 1975.

An extension of the lst edition (by Youden) which discusses the philosophy of collaborative testing and presents AOAC guidelines for planning and conducting a collaborative test.

[160] A. W. Zacherle, A Summary of Federal Quality Assurance Programs Related to Marine Pollution, NOAA, National Marine Pollution Program, 11400 Rockville Pike, Rockville MD 20852.

This report describes the quality assurance practices being followed by seven U. S. government agencies that are engaged in various measurement activities related to marine pollution research. 


\section{TERMINOLOGY USED IN QUALITY ASSURANCE*}

The following contains definitions of 75 terms used most frequently when describing the quality aspects of chemical measurement data. The definitions are reprinted from "Principles of Quality Assurance of Chemical Measurements" [124] and come from various sources, including the author's long association with analytical chemical measurements. They do not necessarily represent official endorsement of the National Bureau of Standards, but rather the author's opinion of appropriateness especially in the context of quality assurance of chemical measurements.

Absolute Method - A method in which characterization is based entirely on physically (absolute) defined standards.

Accreditation - A formal process by which a laboratory is evaluated, with respect to established criteria, for its competence to perform a specified kind(s) of measurement. Also, the decision based upon such a process. When a certificate is issued, the process is often called certification.

Accuracy - The degree of agreement of a measured value with the true or expected value of the quantity of concern.

Aliquant - A divisor that does not divide a sample into a number of equal parts without leaving a remainder; a sample resulting from such a divisor (see also aliquot).

Aliquot - A divisor that divides a sample into a number of equal parts, leaving no remainder; a sample resulting from such a divisor (see also aliquant).

Analyte - The specific component measured in a chemical analysis; also called analate.

Assignable cause - A cause believed to be responsible for an identifiable change of precision or accuracy of a measurement process.

Blank - The measured value obtained when a specified component of a sample is not present during the measurement. In such a case, the measured value/signal for the component is believed to be due to artifacts, hence should be deducted from a measured value to give a net value due to the component contained in a sample. The blank measurement must be made so that the correction process is valid.

Blind Sample - A sample submitted for analysis whose composition is known to the submitter but unknown to the analyst. A blind sample thus is one way to test proficiency of a measurement process.

\footnotetext{
*For other definitions, and particularly those related to industrial quality assurance, see: "Quality Systems Terminology", ANSI/ASQC Standard A3-1978, American National Standards Institute, 1430 Broadway, New York, NY 10018.
} 
Bias - A systematic error inherent in a method or caused by some artifact or idiosyncrasy of the measurement system. Temperature effects and extraction inefficiencies are examples of the first kind. Blanks, contamination, mechanical losses and calibration errors are examples of the latter kinds. Bias may be both positive and negative and several kinds can exist concurrently, so that net bias is all that can be evaluated, except under special conditions.

Bulk sampling - Sampling of a material that does not consist of discrete, identifiable, constant units, but rather of arbitrary, irregular units.

Calibrant - A substance used to calibrate or to establish the analytical response of a measurement system.

Calibration - Comparison of a measurement standard or instrument with another standard or instrument to report or eliminate by adjustment any variation (deviation) in the accuracy of the item being compared.

Cause-Effect Diagram - A graphical representation of the causes that can produce a specified kind of error in measurement. A popular one is the so-called fish bone diagram, first described by Ishikawa, given this name because of its suggestive shape.

Certification - See accreditation.

Central Line - The long-term expected value of a variable displayed on a control chart.

Certified Reference Material (CRM) - A reference material one or more of whose property values are certified by a technically valid procedure, accompanied by or traceable to a certificate or other documentation which is issued by a certifying body.

Certified Value - The value that appears in a certificate as the best estimate of the value for a property of a reference material.

Chance Cause - A cause for variability of a measurement process that occurs unpredictably, for unknown reasons, and believed to happen by chance, alone.

Check Standard - In physical calibration, an artifact measured periodically, the results of which typically are plotted on a control chart to evaluate the measurement process.

Coefficient of Variation - The standard deviation divided by the value of the parameter measured.

Common Cause - A cause of variability of a measurement process, inherent in and common to the process itself, as contrasted to a special cause (def ined).

Comparative Method - A method which is based on the intercomparison of the sample with a chemical standard. 
Confidence Interval - That range of values, calculated from an estimate of the mean and the standard deviation, which is expected to include the population mean with a stated level of confidence. Confidence intervals in the same context also may be calculated for standard deviations, lines, slopes, points.

Control Limit - The limits shown on a control chart beyond which it is highly improbable that a point could lie while the system remains in a state of statistical control.

Control Chart - A graphical plot of test results with respect to time or sequence of measurement together with limits within which they are expected to lie when the system is in a state of statistical control.

Control Sample - A material of known composition that is analyzed concurrently with test samples to evaluate a measurement process (see also Check Standard).

Composite Sample - A sample composed of two or more increments selected to represent a population of interest.

Cross Sensitivity - A quantitative measure of the response obtained for an undesired constituent (interferrent) as compared to that for a constituent of interest.

Detection Limit - The smallest concentration/amount of some component of interest that can be measured by a single measurement with a stated level of confidence.

Double Blind - A sample, known by the submitter but submitted to an analyst in such a way that neither its composition nor its identification as a check sample are known to the latter.

Duplicate Measurement - A second measurement made on the same (or identical) sample of material to assist in the evaluation of measurement variance.

Duplicate Sample - A second sample randomly selected from a population of interest (see also split sample) to assist in the evaluation of sample variance.

Education - Disciplining the mind through instruction or study. Education is general and prepares the mind to react to a variety of situations.

Error - Difference between the true or expected value and the measured value of a quantity or parameter.

Figure of Merit - A performance characteristic of a method believed to be useful when deciding its applicability for a spacific measurement situation. Typical figures of merit include: selectivity; sensitivity; detection limit; precision; bias. 
Good Laboratory Practice (GLP) - An acceptable way to perform some basic operation or activity in a laboratory, that is known or believed to influence the quality of its outputs. GLP's ordinarily are essentially independent of the measurement techniques used.

Good Measurement Practice (GMP) - An acceptable way to perform some operation associated with a specific measurement technique, and which is known or believed to influence the quality of the measurement.

Gross Sample (also called bulk sample, lot sample) - One or more increments of material taken from a larger quantity (lot) of material for assay or record purposes.

Homogeneity - The degree to which a property or substance is randomly distributed throughout a material. Homogeneity depends on the size of the subsample under consideration. Thus a mixture of two minerals may be inhomogeneous at the molecular or atomic level, but homogeneous at the particulate level.

Incrememt - An individual portion of material collected by a single operation of a sampling device, from parts of a lot separated in time or space. Increments may be either tested individually or combined (composited) and tested as a unit.

Individuals - Conceivable constituent parts of a population.

Informational Value - Value of a property, not certified but provided because it is believed to be reliable and to provide information important to the certified material.

Intercalibration - The process, procedures, and activities used to ensure that the several laboratories engaged in a monitoring program can produce compatible data. When compatible data outputs are achieved and this situation is maintained, the laboratories can be said to be intercalibrated.

Laboratory Sample - A sample, intended for testing or analysis, prepared from a gross sample or otherwise obtained. The laboratory sample must retain the composition of the gross sample. Often reduction in particle size is necessary in the course of reducing the quantity.

Limiting Mean - The value approached by the average as the number of measurements, made by a stable measurement process, increases indefinitely.

Limit of Linearity (LOL) - The upper limit of concentration or amount of substance for which incremental additions produce constant increments of response.

Limit of Quantitation (LOQ) - The lower limit of concentration or amount of substance that must be present before a method is considered to provide quantitative results. By convention $L O Q=10 s_{O}$, where $s_{O}$ is the estimate of the standard deviation at the lowest level of measurement. 
Lot - A quantity of bulk material of similar composition whose properties are under study.

Method - An assemblage of measurement techniques and the order in which they are used.

Outlier - A value which appears to deviate markedly from that for other members of the sample in which it occurs.

Pareto Analysis - A statistical approach to ranking assignable causes according to the frequency of occurrence.

Performance Audit - A process to evaluate the proficiency of an analyst/ laboratory by evaluation of the results obtained on known test materials.

Population - A generic term denoting any finite or infinite collection of individual things, objects, or events; in the broadest concept, an aggregate determined by some property that distinguishes things that do and do not belong.

Precision - The degree of mutual agreement characteristic of independent measurements as the result of repeated application of the process under specified conditions. It is concerned with the closeness together of results.

Primary Standard - A substance or artifact, the value of which can be accepted (within specific limits) without question when used to establish the value of the same or related property of another material. Note that the primary standard for one user may have been a secondary standard of another.

Probability - The likelihood of the occurrence of any particular form of an event, estimated as the ratio of the number of ways or times that the event may occur in that form to the total number of ways that it could occur in any form.

Procedure - A set of systematic instructions for using a method of measurement or of sampling or of the steps or operations associated with such.

Protocol - A procedure specified to be used when performing a measurement or related operation, as a condition to obtain results that could be acceptable to the specifier.

Protocol for a Specific Purpose (PSP) - Detailed instructions for the performance of all aspects of a specific measurement program. This is sometimes called a project QA plan.

Quality - An estimation of acceptability or suitability for a given purpose of an object, item, tangible, or intangible thing. 
Quality Assessment - The overall system of activities whose purpose is to provide assurance that the quality control activities are being done effectively. It involves a continuing evaluation of performance of the production system and the quality of the products produced.

Quality Assurance - A system of activities whose purpose is to provide to the producer or user of a product or a service the assurance that it meets defined standards of quality. It consists of two separate but related activities, quality control and quality assessment (defined).

Quality Circle - A small group of individuals with related interests that meets at regular intervals to consider problems or other matters related to the quality of outputs of a process and to the correction of problems or to the improvement of quality.

Quality Control - The overall system of activities whose purpose is to control the quality of a product or service so that it meets the needs of users. The aim is to provide quality that is satisfactory, adequate, dependable, and economic.

Random Sample - A sample selected from a population, using a randomization process.

Reduction - The process of preparing one or more subsamples from a sample.

Reference Material (RM) - A material or substance one or more properties of which are sufficiently well established to be used for the calibration of an apparatus, the assessment of a measurement method, or for the assignment of values to materials.

Reference Method - A method which has been specified as capable, by virtue of recognized accuracy, of providing primary reference data.

Relative Standard Deviation - The coefficient of variation, expressed as a percentage.

Replicate - A counterpart of another, usually referring to an analytical sample or a measurement. It is the general case for which duplicate is the special case consisting of two samples or measurements.

Routine Method - A method used in recurring analytical problems.

Sample - A portion of a population or lot. It may consist of an individual or groups of individuals. It may refer to objects, materials, or to measurements, conceivable as part of a larger group that could have been considered.

Secondary Standard - A standard whose value is based upon comparison with some primary standard. Note that a secondary standard, once its value is established, can become a primary standard for some other user. 
Segment - A specifically demarked portion of a lot, either actual or hypothetical.

Selectivity - The ability of methodology or instrumentation to respond to a desired substance or constituent and not to others. It is sometimes quantified as cross sensitivity, which see.

Sensitivity - Capability of methodology or instrumentation to discriminate between samples having differing concentrations or containing differing amounts of an analate.

Significant Figure - A figure(s) that remains to a number or decimal after the ciphers to the right or left are cancelled.

Special Cause - A cause of variance or bias that is external (not inherent) to the measurement system.

Split Sample - A replicate portion or sub-sample of a total sample obtained in such a manner that it is not believed to differ significantly from other portions of the same sample.

Standard - A substance or material, the properties of which are believed to be known with sufficient accuracy to permit its use to evaluate the same property of another. In chemical measurements, it of ten describes a solution or substance, commonly prepared by the analyst, to establish a calibration curve or the analytical response function of an instrument.

Standard - That which is established by authority, custom, or general consent: as a rule for the measure or judgment of quantity, value, or quality; as a model or example; as an (or even the) acceptable may to conduct a measurement or test.

Standardization - The process whereby the value of a potential standard is fixed by measurement with respect to a standard(s) of known value.

Standard Addition - A method in which small increments of a substance under measurement are added to a sample under test to establish a response function or, by extrapolation, to detemine the amount of a constituent orginally present in the test sample.

Standard Method - A method (or procedure) of test developed by a standardswriting organization, based on consensus opinion or other criteria, and often evaluated for its reliability by a collaborative testing procedure.

Standard Operations Procedure (SOP) - A procedure adopted for repetitive use when performing a specific measurement or sampling operation. It may be a standard method or one developed by the user.

Standard Reference Material - A reference material distributed and certified by the National Bureau of Standards.

Strata - Segments of a lot that may vary with respect to the property under study. 
Subsample - A portion taken from a sample. A laboratory sample may be a subsample of a gross sample; similarly, a test portion may be a subsample of a laboratory sample.

Technique - A physical or chemical principle utilized separately or in combination with other techniques to determine the composition (analysis) of materials.

Test Portion (also called specimen, test specimen, test unit, aliquot) That quantity of a material of proper size for measurement of the property of interest. Test portions may be taken from the gross sample directly, but often preliminary operations, such as mixing or further reduction in particle size, are necessary.

Tolerance Interval - That range of values, calculated from an estimate of the mean and the standard derivation, within which a specified percentage of individual values of population (measurements or sample) are expected to lie with a stated level of confidence.

Traceability - The ability to trace the source of uncertainty of a measurement or a measured value.

Training - Formal or informal instruction designed to provide competence of a specific nature.

Uncertainty - The range of values within which the true value is estimated to lie. It is a best estimate of possible inaccuracy due to both random and systematic error.

Validation - The process by which a sample, measurement method, or a piece of data is deemed to be useful for a specified purpose.

Variance - The value approached by the average of the sum of the squares of deviations of individual measurements from the limiting mean. Mathematically, it may be expressed as

$$
\frac{\sum\left(x_{i}-m\right)^{2}}{n} \rightarrow \sigma^{2} \text { as } n \rightarrow \infty
$$

Ordinarily it cannot be known but only its estimate, $s^{2}$, which is calculated by the expression

$$
s^{2}=\frac{\sum\left(x_{i}-\bar{x}\right)^{2}}{n-1}
$$

Warning Limits - The limits shown on a control chart within which most of the test results are expected to lie (within a $95 \%$ probability) while the system remains in a state of statistical control. 
Youden Plot - A graphical presentation of data, recommended first by $W . J$. Youden, in which the result(s) obtained by a laboratory on one sample is plotted with respect to the result(s) it obtained on a similar sample. It helps in deciding whether discrepant results are due to random or systematic error. 
4. INDEX

Accreditation

Atmospheric analysis

Audits

Blank

Calibration

Certification

Clinical analysis

Control charts

Corrective actions

Definitions

Environmental analysis

Evaluation of laboratories

Food and drug analysis

General

Information management

Marine analysis

Measurement as a process

Measurement programs QA

Methodology

Microbiological analysis

Modeling

Notebooks

Nuclear measurements

Organic trace analysis

Planning
$10,11,17,24,31,61,69,85,105,106,118$

$10,32,35,48,57,102$

53

99,136

$14,46,64,65,75,81,87,127,128$

see Accreditation

$27,33,38,67,112,143$

$7,147,148$

89

6,132

$2,3,5,41,45,98,105,125,127,130,140$

see Accreditation

36

$2,5,26,33,40,43,54,60,68,72,83,95,102$, $109,116,121,123,132,133,141$

113

128,160

$101,110,117,127$

138

$29,42,51,54,62,88,91,127,135,136,139$, $142,152,153,154$

$28,29,149,150$

58

73

8

60

58 
Planning QA programs

Quality assessment

Quality circles

Quality control

Reference materials

Reporting data

Sampling

Sediment analysis

Soil analysis

Spectrographic analysis

Statistical analysis

Terminology

Toxic substance analysis

Traceability

Validation

Water analysis
$1,8,30,45,48,125,132,137$

$23,39,53,98,115,127$

4,44

$1,20,21,25,47,59,74,76,86,135$

$34,70,71,90,92,120,124,127,131,134$

$7,12,25,44,98,102,113$

$66,77,78,79,96,97,111,119,122,144$

126

126

16,19

$9,13,14,15,18,46,80,81,82,84,87,88,89$, $100,103,104,107,146,147,149,150,155,156$, $157,158,159$

$6,28,70,132$

37

$22,63,93,94,101,117$

32, 102, 114, 127, 135

$11,47,50,52,55,61,76,108,115,128$ 
NBS-114A (REV. 2-8C)

U.S. DEPT. OF COMM.

BIBLIOGRAPHIC DATA

SHEET (See instructions)

1. PUBLICATION OR REPORT NO.

NBSIR-86/3352

APRIL 1986

4. TITLE AND SUBTITLE

A COLLECTION OF ABSTRACTS OF SELECTED PUBLICATIONS RELATED TO OQUALITY ASSURANCE OF CHEMICAL MEASUREMENTS

5. $\operatorname{AUTHOR}(S)$

John K. Taylor

6. PERFORMING ORGANIZATION (If joint or other than NBS, see instructions)

7. Contract Grant No.

NATIONAL BUREAU OF STANDARDS

DEPARTMENT OF COMMERCE

WASHINGTON, D.C. 20234

8. Type of Report \& Period Covered

9. SPONSORING ORGANIZATION NAME AND COMPLETE ADDRESS (Street, City, Stote, ZIP)

10. SUPPLEMENTARY NOTES

Document describes a computer program; SF-185, FIPS Software Summary, is attached.

11. ABSTRACT (A 200-word or less factual summary of most significant information. If document includes a significant bibliography or literature survey, mention it here)

This publication contains abstracts of 150 papers selected for their usefulness to analytical chemists, laboratory managers, and quality assurance officials when developing new or improving existing programs or for general guidance in producing reliable analytical chemical measurements. Definitions of some 75 terms used in describing the quality aspects of chemical measurements are included.

12. KEY WORDS (Six to twelve entries; alphabetical order: capitalize only proper names; and separate key words by semicolons) Accuracy of measurements; chemical analysis; chemical terminology; precision of measurements; quality assurance; quality control; reference materials.

13. AVAILABILITY

X] Unlimited

$\square$ For Official Distribution. Do Not Release to NTIS

Order From Superintendent of Documents, U.S. Government Printing Office, Washington, D.C. 20402.

X] Order From National Technical Information Service (NTIS), Springfield, VA. 2216I
14. NO. OF

PRINTED PAGES

51

15. Price

$\$ 11.95$ 


. 